PROCEEDINGS OF THE

AMERICAN MATHEMATICAL SOCIETY

Volume 137, Number 8, August 2009, Pages 2623-2630

S 0002-9939(09)09927-4

Article electronically published on April 7, 2009

\title{
STRONGLY COMPACT NORMAL OPERATORS
}

\author{
MIGUEL LACRUZ AND LUIS RODRÍGUEZ-PIAZZA
}

(Communicated by Marius Junge)

\begin{abstract}
An algebra of bounded linear operators on a Hilbert space is said to be strongly compact if its unit ball is precompact in the strong operator topology, and a bounded linear operator on a Hilbert space is said to be strongly compact if the unital algebra generated by the operator is strongly compact. We show that the position operator on the space of square integrable functions with respect to a finite measure of compact support is strongly compact if and only if the restriction of the measure to the boundary of the polynomially convex hull of its support is purely atomic. This result is applied to construct a strongly compact operator that generates a weakly closed unital algebra that fails to be strongly compact. Also, we construct an operator such that the weakly closed unital algebra generated by the operator is strongly compact but the bicommutant of the operator fails to be a strongly compact algebra. Finally, we prove that a strongly compact operator cannot be strictly cyclic.
\end{abstract}

\section{INTRODUCTION}

Let $\mathcal{B}(H)$ denote the algebra of all bounded linear operators on a complex Hilbert space $H$. A subalgebra $\mathcal{R}$ of $\mathcal{B}(H)$ is said to be strongly compact if its unit ball $\{R \in \mathcal{R}:\|R\| \leq 1\}$ is precompact in the strong operator topology. An operator $T \in \mathcal{B}(H)$ is said to be strongly compact if the algebra with identity generated by $T$ is strongly compact.

This notion was introduced by Lomonosov 4 as a technique to produce invariant subspaces for essentially normal operators on Hilbert spaces. He showed that if $T$ is an essentially normal operator on a Hilbert space such that both its commutant $\{T\}^{\prime}$ and the commutant of its adjoint $\left\{T^{*}\right\}^{\prime}$ fail to be strongly compact algebras, then $T$ has a non-trivial invariant subspace. Moreover, if both the bicommutant of the operator $\{T\}^{\prime \prime}$ and the bicommutant of its adjoint $\left\{T^{*}\right\}^{\prime \prime}$ fail to be strongly compact algebras, then $T$ has a non-trivial hyperinvariant subspace.

Recall that an operator $T$ on a Hilbert space is essentially normal if $T^{*} T-T T^{*}$ is compact. Also, recall that the commutant of a set $\mathcal{S}$ of operators on a Hilbert

Received by the editors July 4, 2008.

2000 Mathematics Subject Classification. Primary 47B07, 47B15, 47L10.

Key words and phrases. Algebras of operators on Hilbert spaces, strongly compact algebras, normal operators, Dirichlet algebras, strictly cyclic operators.

The first author's research was partially supported by Junta de Andalucía under Grant FQM260.

The second author's research was partially supported by Junta de Andalucía under Grant FQM-627.

(C)2009 American Mathematical Society Reverts to public domain 28 years from publication 
space is the set $\mathcal{S}^{\prime}$ of all operators that commute with every element of $\mathcal{S}$. It turns out that $\mathcal{S}^{\prime}$ is a subalgebra of $\mathcal{B}(H)$ and it is closed in the weak operator topology.

Marsalli [5] carried out a classification of operator algebras, and he showed that a weakly closed, self-adjoint algebra of bounded linear operators on a Hilbert space is strongly compact if and only if it can be decomposed as a direct sum of finite dimensional, self-adjoint algebras.

Prunaru 6] showed that if $T$ is a pure hyponormal, essentially normal operator on a Hilbert space, then the commutant of its adjoint $\left\{T^{*}\right\}^{\prime}$ is strongly compact.

The authors and Lomonosov 3 obtained a characterization for strongly compact normal operators in terms of their spectral representation. This characterization is stated below in Theorem 1.1 for the position operator and in Theorem 1.3 for a normal operator on a separable Hilbert space.

Let $\mu$ be a finite measure of compact support defined on the Borel subsets of the complex plane. The position operator $M_{z}$ is defined on $L^{2}(\mu)$ by $\left(M_{z} f\right)(z)=z f(z)$. Thus, $M_{z}$ is a normal operator and its spectrum is the compact set $K=\operatorname{supp}(\mu)$. Let $\pi$ denote the space of polynomials $p(z)$ in one complex variable provided with the norm $\|p\|_{K}=\sup \{|p(z)|: z \in K\}$.

Theorem 1.1. The following conditions are equivalent:

(1) $M_{z}$ is strongly compact on $L^{2}(\mu)$.

(2) The natural embedding $J: \pi \hookrightarrow L^{2}(\mu)$ is compact.

(3) Any bounded sequence in $\pi$ has a subsequence that converges a.e.

Radjavi and Rosenthal [7] say that a common statement of the spectral theorem is the assertion that every normal operator can be represented as an integral with respect to a spectral measure. More precisely,

Theorem 1.2. If $T$ is a normal operator on a Hilbert space, then there exists a unique spectral measure $E$ such that

$$
T=\int \lambda d E_{\lambda} .
$$

If $E$ is the spectral measure associated with a normal operator $T$ on a separable Hilbert space, then there exists a probability measure $\mu$ defined on the Borel subsets of $\mathbb{C}$ such that $E(B)=0$ if and only if $\mu(B)=0$. Such a probability measure is called a control measure for the spectral measure.

Theorem 1.3. Let $T$ be a normal operator on a separable Hilbert space $H$, let $\mu$ be a control measure for the spectral measure associated with $T$, and let $M_{z}$ denote the position operator on $L^{2}(\mu)$. The following conditions are equivalent:

(1) $T$ is strongly compact on $H$.

(2) $M_{z}$ is strongly compact on $L^{2}(\mu)$.

This paper deals with an intrinsic characterization for the strong compactness of the position operator. The characterization, unlike Theorem 1.1 above, is expressed in terms of the measure and not in terms of anything external. This result allows us to construct a strongly compact normal operator that generates a weakly closed unital subalgebra that fails to be strongly compact. Also, we construct an operator such that the weakly closed unital algebra generated by the operator is strongly compact but the bicommutant of the operator fails to be a strongly compact algebra. Finally, we prove that a strongly compact algebra cannot be strictly cyclic and that a strongly compact operator cannot be strictly cyclic. 


\section{The MAIN RESUlT}

Let us start with some definitions before we state the main result. Let $K \subseteq \mathbb{C}$ be a compact set and let $G$ denote the unbounded connected component of $\mathbb{C} \backslash K$. The polynomially convex hull of $K$ is the compact set $\widehat{K}=\mathbb{C} \backslash G$. It is plain that $K \subseteq \widehat{K}$ and that $\mathbb{C} \backslash \widehat{K}$ is connected. If $T$ is a bounded linear operator on a Hilbert space and $K$ is the spectrum of $T$, then $\widehat{K}$ is called the full spectrum of $T$.

Now the ground is prepared to state our intrinsic characterization for the strong compactness of the position operator on $L^{2}(\mu)$.

Theorem 2.1. The following conditions are equivalent:

(1) The position operator is strongly compact on $L^{2}(\mu)$.

(2) The restriction of $\mu$ to the boundary of the polynomially convex hull of its support is a purely atomic measure.

A previous result on Dirichlet algebras is needed for the proof of Theorem 2.1. Let $K \subseteq \mathbb{C}$ be a compact set and let $C(K)$ denote the algebra of all complex continuous functions $f$ on $K$ provided with the norm $\|f\|_{K}=\sup \{|f(z)|: z \in K\}$. The algebra $P(K)$ is the closure in $C(K)$ of the algebra of all complex polynomials. It turns out that $P(\partial \widehat{K})$ is a Dirichlet algebra; that is, for every continuous real function $f$ on $\partial \widehat{K}$ and for every $\varepsilon>0$ there is a complex polynomial $p(z)$ such that

$$
|f(z)-\Re p(z)|<\varepsilon, \quad \text { for all } z \in \partial \widehat{K} .
$$

This is a consequence of the Walsh-Lebesgue Theorem. We refer the reader to the book of Gamelin [1] for the proof of it (cf. Corollary 3.4, p. 37).

Lemma 2.2. If $F_{1}, F_{2}$ are two disjoint compact subsets of $\partial \widehat{K}$, then there is a complex polynomial $p(z)$ such that

(1) $|p(z)| \leq 1, \quad$ for all $z \in K$,

(2) $|p(z)| \geq 3 / 4, \quad$ for all $z \in F_{1}$,

(3) $|p(z)| \leq 1 / 4$, for all $z \in F_{2}$.

Proof of Lemma 2.2, Let $\varepsilon>0$ to be chosen later on, and let $r$ be a continuous real function on $\partial \widehat{K}$ with the property that

(1) $r(z) \leq-\varepsilon, \quad$ for all $z \in \partial \widehat{K}$,

(2) $r(z)=-\varepsilon, \quad$ for all $z \in F_{1}$,

(3) $r(z)=\log (1 / 8), \quad$ for all $z \in F_{2}$.

Since $P(\partial \widehat{K})$ is a Dirichlet algebra, there is a complex polynomial $q(z)$ such that

$$
|r(z)-\Re q(z)|<\varepsilon, \quad \text { for all } z \in \partial \widehat{K} .
$$

Therefore, the following inequalities are satisfied:
(1) $\Re q(z)<0$,
for all $z \in \partial \widehat{K}$,
(2) $\Re q(z) \geq-2 \varepsilon$
for all $z \in F_{1}$,
(3) $\Re q(z) \leq \log (1 / 8)+\varepsilon, \quad$ for all $z \in F_{2}$.

Now consider the entire function $f(z)=\exp q(z)$ and notice that $|f(z)|=\exp \Re q(z)$. Thus, the above inequalities are equivalent to
(1) $|f(z)|<1$, for all $z \in \partial \widehat{K}$,
(2) $|f(z)| \geq \exp (-2 \varepsilon), \quad$ for all $z \in F_{1}$,
(3) $|f(z)| \leq \exp (\varepsilon) / 8, \quad$ for all $z \in F_{2}$. 
Since $f$ is an entire function, thanks to the maximum modulus principle, the set $\partial \widehat{K}$ can be replaced by the set $K$ in the first inequality. Now choose $\varepsilon>0$ so small that $\exp (2 \varepsilon)<4 / 3$. Thus, the former inequalities lead to

(1) $|f(z)|<1, \quad$ for all $z \in K$,

(2) $|f(z)|>3 / 4$, for all $z \in F_{1}$,

(3) $|f(z)|<1 / 4$, for all $z \in F_{2}$.

Finally, the Taylor series of $f$ converges to $f$ uniformly on compact sets, so that we can take the desired polynomial $p(z)$ to be a partial sum of a suitable degree.

Proof of Theorem 2.1. Consider the position operator $M_{z}$ defined on $L^{2}(\mu)$ by the expression $\left(M_{z} f\right)(z)=z f(z)$. We mentioned earlier that $M_{z}$ is a normal operator whose spectrum is the compact set $K=\operatorname{supp}(\mu)$.

Assume that the restriction of $\mu$ to $\partial \widehat{K}$ is not a purely atomic measure, so that there is an $\varepsilon>0$ and there are two sequences $\left(F_{1}^{n}\right),\left(F_{2}^{n}\right)$ of compact subsets of $\partial \widehat{K}$ such that $F_{1}^{n} \cap F_{2}^{n}=\varnothing$, for all $n$, and $\mu\left(F_{1}^{n} \cap F_{2}^{m}\right) \geq \varepsilon$, for all $n \neq m$. We must show that $M_{z}$ fails to be a strongly compact operator. Apply Lemma 2.2 to get a sequence of complex polynomials $\left(p_{n}\right)$ with the following properties:

(1) $\left|p_{n}(z)\right| \leq 1, \quad$ for all $z \in K$,

(2) $\left|p_{n}(z)\right| \geq 3 / 4, \quad$ for all $z \in F_{1}^{n}$,

(3) $\left|p_{n}(z)\right| \leq 1 / 4, \quad$ for all $z \in F_{2}^{n}$.

We have $\left\|p_{n}\right\|_{K} \leq 1$ for every $n$. Put $F_{n m}=\left(F_{1}^{n} \cap F_{2}^{m}\right) \cup\left(F_{1}^{m} \cap F_{2}^{n}\right)$ for $n \neq m$, and notice that $\mu\left(F_{n m}\right) \geq 2 \varepsilon$ and $\left|p_{n}(z)-p_{m}(z)\right| \geq 1 / 2$ for all $z \in F_{n m}$. Hence,

$$
\left\|p_{n}-p_{m}\right\|_{L^{2}(\mu)}^{2} \geq \int_{F_{n m}}\left|p_{n}(z)-p_{m}(z)\right|^{2} d \mu(z) \geq \frac{\varepsilon}{2},
$$

so that the sequence $\left(p_{n}\right)$ is not relatively compact in $L^{2}(\mu)$ and it follows from Theorem 1.1 that $M_{z}$ fails to be a strongly compact operator.

Now, assume that the restriction of $\mu$ to $\partial \widehat{K}$ is a purely atomic measure, so that there is a countable set $C \subseteq \partial \widehat{K}$ such that $\mu(\partial \widehat{K} \backslash C)=0$. Consider a sequence $\left(p_{n}\right)$ of polynomials with $\left\|p_{n}\right\|_{K} \leq 1$ for all $n$. The maximum modulus principle gives $\left\|p_{n}\right\|_{\widehat{K}} \leq 1$ for all $n$. Notice that there is a decomposition $\widehat{K}=\operatorname{int}(\widehat{K}) \cup \partial \widehat{K}$. Apply Montel's Theorem to obtain a subsequence $\left(p_{n_{j}}\right)$ that converges uniformly on the compact subsets of the interior of $\widehat{K}$. Finally, $\left\|p_{n_{j}}\right\|_{C} \leq 1$ for all $j$, and since $C$ is a countable set, a diagonal procedure allows us to extract from $\left(p_{n_{j}}\right)$ another subsequence $\left(p_{n_{j_{k}}}\right)$ that converges pointwise on $C$. It is clear that $\left(p_{n_{j_{k}}}\right)$ converges almost everywhere on $K$ with respect to $\mu$. Now it follows from Theorem 1.1 that $M_{z}$ is a strongly compact operator.

As an application of Theorem 2.1 we provide an example of a strongly compact operator that generates a weakly closed algebra that fails to be strongly compact.

Example 2.3. Let $\left(z_{k}\right)$ be a sequence of complex numbers dense in the unit circle, let $\delta_{z_{k}}$ denote the Dirac measure at $z_{k}$, and let $\lambda$ be the normalized Lebesgue measure on the unit interval. Consider the measure $\mu$ defined on the Borel subsets of the complex plane by the expression

$$
\mu(B)=\lambda(B \cap[0,1])+\sum_{k=1}^{\infty} \frac{1}{2^{k}} \delta_{z_{k}}(B) .
$$


It is clear that the support of $\mu$ is the compact set $K=[0,1] \cup \mathbb{T}$. Hence, $\widehat{K}=\overline{\mathbb{D}}$, so that $\partial \widehat{K}=\mathbb{T}$. Since the restriction of $\mu$ to $\mathbb{T}$ is a purely atomic measure, it follows from Theorem 1.1 that the position operator $M_{z}$ is strongly compact on $L^{2}(\mu)$.

We now consider the weakly closed unital algebra $\mathcal{R}_{\sigma}$ generated by $M_{z}$; that is, $\mathcal{R}_{\sigma}$ is the closure in the weak operator topology of $\mathcal{R}=\left\{p\left(M_{z}\right): p\right.$ is a polynomial $\}$. We identify every function $\varphi \in L^{\infty}(\mu)$ with the operator $M_{\varphi}$ of multiplication by $\varphi$ defined on $L^{2}(\mu)$ by the expression $\left(M_{\varphi} f\right)(z)=\varphi(z) f(z)$. It is a well-known fact that $L^{\infty}(\mu)$ is a maximal abelian subalgebra of $\mathcal{B}\left(L^{2}(\mu)\right)$ and therefore it is closed in the weak operator topology. See the book of Radjavi and Rosenthal [7] for the proof of this result (cf. Corollary 1.21, p. 21).

We claim that $\mathcal{R}_{\sigma}=L^{\infty}(\mu)$. Notice that the weak operator topology agrees on $L^{\infty}(\mu)$ with the weak* topology. Also, the algebra $\pi$ of all complex polynomials is identified with the algebra $\mathcal{R}$. Thus, we need to show that $\pi$ is a dense subspace of $L^{\infty}(\mu)$ in the weak* topology. The Hahn-Banach Theorem comes to our rescue. Take any function $f \in L^{1}(\mu)$ with the property that

$$
\int_{K} f p d \mu=0 \quad \text { for all } p \in \pi \text {. }
$$

We have to prove that $f=0$ almost everywhere with respect to $\mu$. Fix $1 \leq k<\infty$ and let us show that $f\left(z_{k}\right)=0$. Define a sequence of polynomials $\left(p_{n}\right)$ by

$$
p_{n}(z)=\left(\frac{z+z_{k}}{2}\right)^{n}
$$

and notice that the following properties are satisfied:

(1) $\left|p_{n}(z)\right| \leq 1 \quad$ for all $z \in \overline{\mathbb{D}}$,

(2) $\lim p_{n}(z)=0$ for all $z \in \overline{\mathbb{D}} \backslash\left\{z_{k}\right\}$,

(3) $p_{n}\left(z_{k}\right)=1 \quad$ for all $n \geq 1$.

Apply the bounded convergence theorem to arrive at the conclusion that

$$
\mu\left(\left\{z_{k}\right\}\right) f\left(z_{k}\right)=\lim _{n \rightarrow \infty} \int_{K} f p_{n} d \mu=0,
$$

so that $f\left(z_{k}\right)=0$, as we wanted. Hence, $f=0$ almost everywhere on $\mathbb{T}$, and we get

$$
\int_{0}^{1} f p d \lambda=\int_{K} f p d \mu=0 \quad \text { for all } p \in \pi
$$

Now the Weierstrass approximation theorem leads to

$$
\int_{0}^{1} f g d \lambda=0 \quad \text { for all } g \in C[0,1]
$$

so that $f=0$ almost everywhere on $[0,1]$. Therefore, $f=0$ almost everywhere with respect to $\mu$, and the proof of our claim that $\mathcal{R}_{\sigma}=L^{\infty}(\mu)$ is over.

We finally show that $\mathcal{R}_{\sigma}$ fails to be a strongly compact algebra. Define a sequence of functions $\left(\varphi_{n}\right)$ in $L^{\infty}(\mu)$ by the expression $\varphi_{n}(z)=\exp (2 \pi i n z)$, if $z \in[0,1]$, and $\varphi_{n}(z)=0$, otherwise. Notice that $\left\|M_{\varphi_{n}}\right\|=\left\|\varphi_{n}\right\|_{\infty}=1$, so that the sequence of 
operators $\left(M_{\varphi_{n}}\right)$ lies inside the unit ball of $\mathcal{R}_{\sigma}$. Moreover, if $n \neq m$, then

$$
\begin{gathered}
\left\|M_{\varphi_{n}} 1-M_{\varphi_{m}} 1\right\|_{2}^{2}=\int_{K}\left|\varphi_{n}-\varphi_{m}\right|^{2} d \mu \\
\geq \int_{0}^{1}\left|e^{2 \pi i n t}-e^{2 \pi i m t}\right|^{2} d \lambda(t)=2,
\end{gathered}
$$

so that $\left(M_{\varphi_{n}} 1\right)$ is not relatively compact in $L^{2}(\mu)$, and we conclude that $\left(M_{\varphi_{n}}\right)$ is not relatively compact in the strong operator topology.

Now we construct an example of an operator such that the weakly closed algebra generated by the operator is strongly compact but the bicommutant of the operator fails to be a strongly compact algebra.

Example 2.4. Consider the normalized Lebesgue area measure on the unit disc and consider the position operator $M_{z}$ on the space $L^{2}(\mathbb{D})$. Also, let $\mathcal{R}_{\sigma}$ denote the weakly closed algebra generated by $M_{z}$, that is, the closure in the weak operator topology of the algebra $\mathcal{R}=\left\{p\left(M_{z}\right): p\right.$ is a polynomial $\}$.

We identify every function $\varphi$ in $L^{\infty}(\mathbb{D})$ or in $H^{\infty}(\mathbb{D})$ with the multiplication operator $M_{\varphi}$ defined on $L^{2}(\mathbb{D})$ by the expression $\left(M_{\varphi} f\right)(z)=\varphi(z) f(z)$, and under this identification, we regard $L^{\infty}(\mathbb{D})$ and $H^{\infty}(\mathbb{D})$ as two subalgebras of $\mathcal{B}\left(L^{2}(\mathbb{D})\right)$. Notice that the weak* topology agrees on $L^{\infty}(\mathbb{D})$ with the weak operator topology.

Montel's Theorem gives us that the unit ball of $H^{\infty}(\mathbb{D})$ is a compact subset of $L^{\infty}(\mathbb{D})$ in the weak* topology. A consequence of the Banach-Dieudonné Theorem is the fact that a subspace of a dual Banach space is closed in the weak* topology if and only if its unit ball is compact in the weak* topology. We refer to the book of Horváth 2 for the proof of this fact. Therefore, $H^{\infty}(\mathbb{D})$ is a closed subspace of $L^{\infty}(\mathbb{D})$ in the weak* topology. Hence, $H^{\infty}(\mathbb{D})$ is a weakly closed algebra.

Now $\mathcal{R} \subseteq H^{\infty}(\mathbb{D})$, so that $\mathcal{R}_{\sigma} \subseteq H^{\infty}(\mathbb{D})$, and since the space of polynomials is dense in $H^{\infty}(\mathbb{D})$ with respect to the weak* topology, it follows that $\mathcal{R}_{\sigma}=H^{\infty}(\mathbb{D})$. In order to prove that $\mathcal{R}_{\sigma}$ is a strongly compact algebra, we need to show that, for every $f \in L^{2}(\mathbb{D})$, the set $\left\{\varphi f: \varphi \in H^{\infty}(\mathbb{D}),\|\varphi\|_{\infty} \leq 1\right\}$ is precompact in $L^{2}(\mathbb{D})$. A straightforward application of Montel's Theorem and the Bounded Convergence Theorem shows that this set is indeed compact in $L^{2}(\mathbb{D})$.

Let us compute the bicommutant $\left\{M_{z}\right\}^{\prime \prime}$ of the position operator. First of all, it is obvious that $\left\{M_{z}\right\}^{\prime} \supseteq L^{\infty}(\mathbb{D})$. We claim that in fact $\left\{M_{z}\right\}^{\prime}=L^{\infty}(\mathbb{D})$. Take an operator $T \in\left\{M_{z}\right\}^{\prime}$, that is, $T M_{z}=M_{z} T$. It follows from Fuglede's Theorem that $T M_{\bar{z}}=M_{\bar{z}} T$, which implies that $T M_{p(z, \bar{z})}=M_{p(z, \bar{z})} T$ for every polynomial $p$ in two variables. Apply the Stone-Weierstrass Theorem to see that $T M_{\varphi}=M_{\varphi} T$ for every $\varphi \in C(\overline{\mathbb{D}})$, and use weak* density to conclude that the same identity holds for every $\varphi \in L^{\infty}(\mathbb{D})$. This means that $T \in L^{\infty}(\mathbb{D})^{\prime}$, the commutant of $L^{\infty}(\mathbb{D})$. The algebra $L^{\infty}(\mathbb{D})$ is maximal abelian, as mentioned in Example 2.3. so that $L^{\infty}(\mathbb{D})^{\prime}=L^{\infty}(\mathbb{D})$. Hence, $T \in L^{\infty}(\mathbb{D})$ and the proof of our claim is complete. Finally, notice that $\left\{M_{z}\right\}^{\prime \prime}=L^{\infty}(\mathbb{D})$ because, once again, the algebra $L^{\infty}(\mathbb{D})$ is maximal abelian.

The conclusion is that the bicommutant $\left\{M_{z}\right\}^{\prime \prime}$ of the position operator fails to be a strongly compact algebra, because the same argument of Example 2.3 with the functions $\varphi_{n}(z)=(z /|z|)^{n}$ shows that the algebra $L^{\infty}(\mathbb{D})$ fails to be strongly compact. This is a particular instance of the more general fact that the natural embedding $L^{\infty}(\mu) \hookrightarrow L^{2}(\mu)$ is not a compact operator whenever $\mu$ is a non-purely atomic, finite measure. 


\section{STRICTLY CYCLIC OPERATORS}

A subalgebra $\mathcal{R}$ of $\mathcal{B}(H)$ is said to be strictly cyclic if there is a vector $x_{0} \in H$ such that $\left\{R x_{0}: R \in \mathcal{R}\right\}=H$. An operator $T \in \mathcal{B}(H)$ is said to be strictly cyclic if the weakly closed unital algebra generated by $T$ is strictly cyclic. This notion has been studied by many authors. We refer to the survey of Allen Shields 8 for references and background on strictly cyclic operators.

The aim of this section is to show that a strongly compact operator on an infinite dimensional Hilbert space cannot be strictly cyclic. Let us introduce some notation. If $\mathcal{R}$ is a subalgebra of $\mathcal{B}(H)$, then $\mathcal{R}_{u}$ denotes the closure of $\mathcal{R}$ in the operator norm, and $\mathcal{R}_{\sigma}$ denotes the closure of $\mathcal{R}$ in the weak operator topology.

Theorem 3.1. If an algebra $\mathcal{R}$ of operators on an infinite dimensional Hilbert space $H$ is strongly compact, then $\mathcal{R}$ is not strictly cyclic.

Proof. First notice that if $\mathcal{R}$ is strongly compact, then $\mathcal{R}_{u}$ is also strongly compact. Given any vector $x_{0} \in H$, the set $\left\{R x_{0}: R \in \mathcal{R}_{u},\|R\| \leq 1\right\}$ is precompact in $H$, and since $H$ is infinite dimensional, it has empty interior. It follows that the set $\left\{R x_{0}: R \in \mathcal{R}_{u}\right\}$ also has empty interior. Therefore $\left\{R x_{0}: R \in \mathcal{R}\right\} \varsubsetneqq H$; that is, the algebra $\mathcal{R}$ fails to be strictly cyclic.

Theorem 3.2. If $T$ is a strongly compact operator on an infinite dimensional Hilbert space, then $T$ is not strictly cyclic.

Proof. The assumption is that $\mathcal{R}=\{p(T): p$ is a polynomial $\}$ is strongly compact. In view of Theorem 3.1, it is tempting to claim that $\mathcal{R}_{\sigma}$ is also strongly compact and to conclude that $\mathcal{R}_{\sigma}$ fails to be strictly cyclic. However, this is misleading since the algebra $\mathcal{R}_{\sigma}$ may not be strongly compact, just as in Example 2.3.

We proceed by contradiction. We assume that the algebra $\mathcal{R}_{\sigma}$ is strictly cyclic. Since $\mathcal{R}_{\sigma}$ is abelian, it follows from a result of Allen Shields 8 , that the strong operator topology agrees on $\mathcal{R}_{\sigma}$ with the norm topology. Now, the unit ball of $\mathcal{R}$ is precompact in the strong operator topology, so that it is also precompact in the norm topology. Hence, $\operatorname{dim} \mathcal{R}<\infty$, so that $\operatorname{dim} \mathcal{R}_{\sigma}<\infty$, and since the algebra $\mathcal{R}_{\sigma}$ is strictly cyclic, it follows that $\operatorname{dim} H<\infty$, a contradiction.

\section{REFERENCES}

[1] Theodore W. Gamelin. Uniform algebras. Prentice-Hall Inc., Englewood Cliffs, N.J., 1969. MR0410387 (53:14137)

[2] John Horváth. Topological vector spaces and distributions. Vol. I. Addison-Wesley Publishing Co., Reading, Mass.-London-Don Mills, Ont., 1966. MR0205028 (34:4863)

[3] Miguel Lacruz, Victor Lomonosov, and Luis Rodríguez-Piazza. Strongly compact algebras. RACSAM Rev. R. Acad. Cienc. Exactas Fís. Nat. Ser. A Mat., 100(1-2):191-207, 2006. MR2267409 (2008b:47032)

[4] V. I. Lomonosov. A construction of an intertwining operator. Funktsional. Anal. i Prilozhen., 14(1):67-68, 1980. MR565106 (81k:47032)

[5] Michael Marsalli. A classification of operator algebras. J. Operator Theory, 24(1):155-163, 1990. MR 1086551(92e:47076)

[6] Bebe Prunaru. On the commutant of hyponormal operators. Proc. Amer. Math. Soc., 124(11):3411-3412, 1996. MR1342042 (97a:47033) 
[7] Heydar Radjavi and Peter Rosenthal. Invariant subspaces. Ergebnisse der Mathematik und ihrer Grenzgebiete, Band 77, Springer-Verlag, New York, 1973. MR0367682 (51:3924)

[8] Allen L. Shields. Weighted shift operators and analytic function theory. In Topics in operator theory, pages 49-128. Math. Surveys, No. 13. Amer. Math. Soc., Providence, R.I., 1974. MR0361899 (50:14341)

Departamento de Análisis Matemático, Facultad de Matemáticas, Universidad de Sevilla, Apartado de Correos 1160, 41080 Sevilla, Spain

E-mail address: lacruz@us.es

Departamento de Análisis Matemático, Facultad de Matemáticas, Universidad de Sevilla, Apartado de Correos 1160, 41080 Sevilla, Spain

E-mail address: piazza@us.es 